\title{
Mortalidade infantil e orçamento público. Desafios na gestão do sistema de saúde no Território Litoral Sul da Bahia, Brasil*
}

\author{
Mortalidad infantil y presupuesto público. Desafíos en la gestión \\ del sistema de salud en el Territorio Litoral Sur de Bahía, Brasil
}

\section{Infant mortality and public budget. Challenges in the management of the health system in the Southern Coastal Territory of Bahia, Brazil}

Recepção: 18 de Agosto de 2019. Aprovação: 10 de Fevereiro de 2020. Publicação: 1 de Outubro de 2020.

DOI: https://doi.org/10.11144/Javeriana.rgps19.miop

\author{
Alciene Pereira da Silva \\ Universidade Federal da Bahia, Brasil \\ ORCID: https://orcid.org/0000-0001-8047-5994 \\ Roque Pinto ${ }^{a}$ \\ Universidade Estadual de Santa Cruz, Brasil \\ ORCID: https://orcid.org/0000-0001-5347-0901 \\ Lacita Menezes Skalinski \\ Universidade Estadual de Santa Cruz, Brasil \\ ORCID: https://orcid.org/0000-0002-6172-0672 \\ Alisson Jadavi Pereira da Silva \\ Universidade Federal do Recôncavo da Bahia, Brasil \\ ORCID: https://orcid.org/0000-0002-1720-4216 \\ Beatriz Barbosa Pinto \\ Universidade Estadual de Santa Cruz, Brasil \\ ORCID: https://orcid.org/0000-0002-5945-204X \\ Ângelo Oliveira Gonçalves \\ Universidade Estadual de Santa Cruz, Brasil \\ ORCID: https://orcid.org/0000-0003-3151-4875
}

\begin{abstract}
Para citar este artigo Silva AP, Pinto R, Skalinski LM, Silva ALP, Pinto BB, Gonçalves AO. Mortalidade infantil e orçamento público. Desafios na gestão do sistema de saúde no Território Litoral Sul da Bahia, Brasil. Rev Gerenc Polit Salud. 2020;19. https://doi.org/10.11144/Javeriana.rgps19.miop
\end{abstract}

a Autor para correspondência. E-mail: roquepintosantos@gmail.com 


\section{Resumo}

Este trabalho pretende discutir como o orçamento público e o modelo da atenção básica em saúde pública podem afetar as taxas de mortalidade infantil em um dado território. A investigação foi realizada no Território Litoral Sul da Bahia, no Estado da Bahia, região Nordeste do Brasil. Trata-se de uma pesquisa quantitativa e descritiva, baseada em dados secundários referentes ao período 2006-2015, coletados entre 2017 e 2018, em bancos de dados públicos oficiais brasileiros. Verificou-se que embora a taxa média de mortalidade infantil tenha caído gradativamente a partir de 2006 no território investigado, ela continua maior do que a média nacional em todo o período pesquisado. Em 2006, a taxa média do território foi de $35,53 \%$, e decresceu até $18,56 \%$ em 2015 . Contudo, em todo o período a taxa média permaneceu maior do que a média brasileira, que é de $18 \%$. Os índices de mortalidade infantil têm forte relação com fatores sociais, econômicos e ambientais. No caso investigado, estão associados à fragmentação e a fragilidade das redes públicas de serviços de saúde nos municípios da região.

Palavras-chave: Mortalidade infantil, epidemiologia, financiamento governamental, Brasil.

\section{Resumen}

Este trabajo pretende discutir cómo el presupuesto público y el modelo de atención básica en salud pública pueden afectar las tasas de mortalidad infantil en un determinado territorio. La investigación fue realizada en el Territorio Litoral Sur de Bahía, en el Estado de Bahía, región Nordeste de Brasil. Se trata de una investigación cuantitativa y descriptiva, basada en datos secundarios referentes al período 2006-2015, recogidos entre 2017 y 2018, en bancos de datos públicos oficiales brasileños. Se verificó que, aunque la tasa media de mortalidad infantil cayó gradualmente a partir de 2006 en el territorio, sigue siendo mayor que la media nacional en todo el período investigado. En 2006, la tasa media del territorio fue del 35,53\%, y disminuyó hasta el 18,56\% en 2015. Sin embargo, en todo el período la tasa media fue superior a la media brasileña, que fue del 18\%. Los índices de mortalidad infantil tienen una fuerte relación con factores sociales, económicos y ambientales. En el caso investigado, están asociados a la fragmentación y la fragilidad de las redes públicas de servicios de salud en los municipios de la región.

Palabras clave: Mortalidad infantil, epidemiología, financiación gubernamental, Brasil.

\section{Abstract}

This paper aims to discuss how the public budget and the model of basic attention in public health could affect the infant mortality rates in a certain territory. The research was carried out in the Southern Coastal Territory of Bahia, in the State of Bahia, Northeast Region of Brazil. It is a quantitative and descriptive investigation, based on secondary data referring to the period from 2006 to 2015, collected between 2017 and 2018 in official Brazilian public data banks. It was verified that, although the average rate of infant mortality fell gradually from 2006 in the territory, it is still higher than the national average within the investigated period. In 2006, the average rate of the territory was $35.53 \%$ and decreased to $18.56 \%$ in 2015 . However, throughout the period the average rate remained higher than the Brazilian average, which is $18 \%$. Infant mortality rates have a strong relationship with social, economic, and environmental factors. In the case investigated, they are associated with the fragmentation and fragility of public health service networks in the municipalities of the region.

Keywords: Infant mortality, epidemiology, government financing, Brazil. 


\section{Introdução}

Os indicadores de saúde são instrumentos indispensáveis para definir prioridades nas políticas públicas, planejar ações e serviços, monitorar a situação da saúde e avaliar o desempenho do sistema de saúde como um todo. Estas métricas são importantes na sistematização de informações necessárias para o planejamento e a implementação das ações em saúde pública com eficiência e eficácia.

Em decorrência da grande vulnerabilidade biológica apesentada pelas crianças com menos de um ano de idade, a mortalidade nessa faixa etária é considerada como um parâmetro das condições de vida de uma população. Esta medição específica permite entrever mais claramente as relações entre as doenças e suas determinantes sociais, especialmente nas questões relativas às desigualdades socioeconômicas e seus desdobramentos antrópicos (1-3).

Dados da Organização Mundial da Saúde destacam que menos da metade da população mundial recebe atualmente todos os serviços de saúde essenciais. Em 2010, por exemplo, quase 100 milhões de pessoas foram levadas à pobreza extrema por terem que pagar pelos serviços de saúde. No ano de 2016, 15 mil crianças menores de cinco anos morreram por dia no mundo (4).

A mortalidade infantil está intimamente relacionada às condições de saneamento, nutrição, vacinação e qualidade da atenção prestada à criança. É por isto que a participação dos serviços de Atenção Básica e da Estratégia Saúde da Família ganha destaque. Estes aproximam a assistência realizada à população da sua realidade, reconhecendo situações de risco e intervindo sobre elas (5).

Nessa perspectiva, este trabalho tem como objetivo discutir como o orçamento público e o modelo da Atenção Básica em saúde pública podem afetar as taxas de mortalidade infantil em um dado território. Observou-se, para este fito, o lapso temporal de dez anos, no Território Litoral Sul do Estado da Bahia, na região nordeste do Brasil.

\section{Metodologia}

Trata-se de uma pesquisa de natureza descritiva e quantitativa. É quantitativa, pois utiliza dados mensuráveis (mortalidade infantil e financiamento) para a apresentação do problema de estudo, e descritiva por limitar-se à análise das relações entre essas duas variáveis. Este trabalho descreve, analisa e interpreta dados temporais e espaciais de mortalidade em menores de um ano de vida representativos de diferentes municípios. De esta forma, pretende-se investigar se existe uma possível relação desta variável com fatores políticos, sociais e ambientais, com especial ênfase no financiamento da atenção básica em saúde.

Para esta finalidade coletou-se, no período entre 2017 e 2018, uma série de dados históricos anuais de mortalidade em menores de um ano de vida (Sistema de Informação de Mortalidade), bem como dados de financiamento da Atenção Básica em saúde (Sistema de Informações sobre Orçamentos Públicos em Saúde - SIOPS). Estes dados foram obtidos no site do Departamento 
de Informática do Sistema Único de Saúde (DATASUS), e do Instituto Brasileiro de Geografia e Estatística (IBGE) (6-8).

Para calcular a taxa de mortalidade infantil foi utilizado o número de óbitos em menores de um ano, dividido pela população de nascidos vivos no mesmo ano por município de residência da mãe, nos municípios pertencentes ao Território Litoral Sul da Bahia. A coleta destes dados seguiu o seguinte procedimento:

1. Coleta dos dados de mortalidade em menores de um ano de vida: no site do DATASUS buscou-se a opção "Estatísticas Vitais - Mortalidade e Nascidos Vivos" escolheu-se a Unidade Federada (Estado da Bahia) e o ano. Foi feito o levantamento de cada cidade do Território, ano a ano (6).

2. Para os dados relativos aos recursos de financiamento da saúde foi utilizada a plataforma "Sistema de Informações sobre Orçamentos Públicos em Saúde (SIOPS)" do Ministério da Saúde do Brasil (7). Na plataforma selecionou-se a opção "conhecer situação do seu município/estado". A partir desta janela foi escolhido o "tipo de ente federado" (município) e o "Estado" (Bahia). Foram coletados os recursos financeiros da atenção básica per capita/ano de cada município.

3. Para o rendimento médio trabalhador em cada município usou-se o sistema IBGECidades. Para o acesso deve-se informar o período temporal, o estado e o município do país (8). As coletas e os cálculos estatísticos foram feitos usando planilha do Excel.

4. Os dados obtidos de mortalidade e financiamento da Atenção Básica se referem a um período de dez anos (2006-2015). Este lapso temporal foi delimitado na investigação porque corresponde ao período disponível no sistema DATASUS até o momento da coleta.

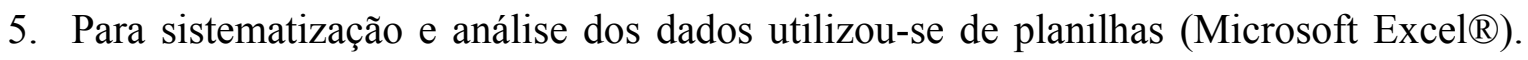
Analisou-se a variação anual absoluta das taxas de mortalidade em menores de um ano de vida, no tempo e no espaço (entre os municípios) e calculou-se a taxa média aritmética de mortalidade referente ao período de dez anos (2006-2015) para cada município e para todo território do estudo.

O terreno de investigação foi o Território Litoral Sul da Bahia, localizado no Estado da Bahia, região nordeste do Brasil. Esta classificação territorial se define segundo o conjunto de características geomorfológicas, ecológicas e antrópicas relativas aos municípios circunscritos a uma área de 14,6 mil Km.. Este território engloba uma população estimada de cerca de 800 mil habitantes, com $75 \%$ dos residentes moradores na área urbana $(8)^{1}$.

Em relação à dimensão ética de pesquisa, por se tratar de uma investigação que utilizou dados secundários disponíveis por fontes oficiais e públicas, não se fez necessário a submissão do protocolo de pesquisa ao Sistema CEP/CONEP (rede brasileira de comitês de ética em pesquisa que são as instâncias institucionais que regulam a dimensão ética em pesquisas científicas no país). 


\section{Resultados}

Ao analisar a mortalidade infantil no Território Litoral Sul identificou-se que no ano 2000 este indicador alcançava 61 crianças/1.000 nascidas vivas. Já entre 2001 e 2010, se reduziu para 22 crianças / 1.000 nascidas vivas. Entretanto, a partir de 2011 a mortalidade infantil aumentou para 38 crianças $/ 1.000$ nascidas vivas.

A figura 1 demonstra a taxa média de mortalidade infantil nos municípios, entre os anos de 2006 a 2015. Apesar da taxa cair gradativamente a partir de 2006, continuou maior que a média nacional (18\%) em todo período pesquisado. Em 2006, a taxa teve seu pico e oscilou no decorrer dos anos. A taxa foi mais alta em 2006 (35\%) quando comparada ao nordeste (31\%) e à média nacional $(18 \%)$ em todo período pesquisado.

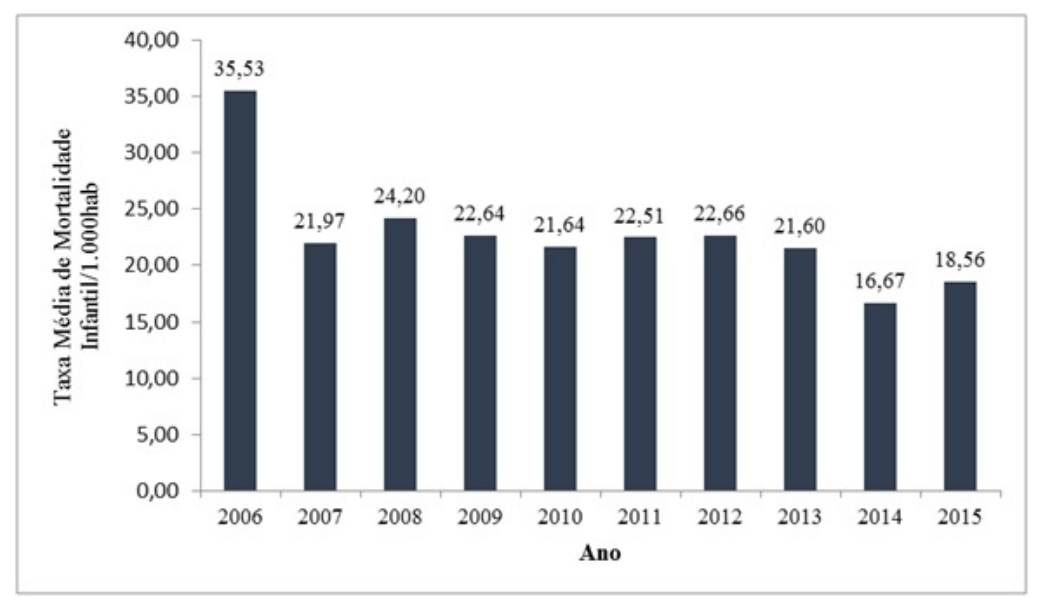

Figura 1 Taxa Média de Mortalidade Infantil /1.000hab

em municípios do Litoral Sul, Bahia, 2006 a 2015 Fonte: Ministério da Saúde, DATASUS, 2017.

Verificou-se que $81 \%$ dos municípios que compõem o Território Litoral Sul da Bahia têm a taxa de mortalidade infantil acima da média nacional. A variação deste indicador pode ser relacionada com fatores sociais, econômicos, ambientais e a fragmentação e fragilidade das redes de serviços entre os municípios consorciados na região. Na tabela 1 pôde-se identificar esta fragilidade em nível municipal, visto que os dois polos de serviços de saúde do Território (Ilhéus e Itabuna) apresentaram taxa média de mortalidade semelhante ou maior do que municípios com menor demografia e menor arrecadação de impostos. 
Alciene Pereira da Silva/ Roque Pinto/ Lacita Menezes Skalinski/ Alisson Jadavi Pereira da Silva/ et ai.

Tabela 1 Taxa média de mortalidade infantil no Território Litoral Sul da Bahia, 2006 a 2015

\begin{tabular}{|c|c|c|c|}
\hline Municipio & Índice & Municipio & İndice \\
\hline Almadina & 14,63 & Itaju do Colônia & 35,17 \\
\hline Arataca & 15,00 & Itajuípe & 23,09 \\
\hline Aurelino Leal & 24,51 & Itapé & 17,69 \\
\hline Barro Preto & 26,14 & Itapitanga & 20,71 \\
\hline Buerarema & 23,75 & Jussari & 14,97 \\
\hline Camacan & 24,86 & Maraú & 20,51 \\
\hline Coaraci & 24,28 & Mascote & 14,77 \\
\hline Canavieiras & 15,78 & Pau Brasil & 19,14 \\
\hline Floresta Azul & 22,26 & Santa Luzia & 13,67 \\
\hline Ibicaraí & 20,01 & São José da Vitória & 16,68 \\
\hline Ibirapitanga & 23,01 & Ubaitaba & 20,49 \\
\hline Ilhéus & 21,12 & Una & 21,71 \\
\hline Itabuna & 22,04 & Uruçuca & 18,58 \\
\hline Itacaré & 20,63 & & \\
\hline
\end{tabular}

Fonte: Ministério da Saúde, DATASUS, 2017.

Na figura 2 é apresentado o mapeamento por Sistema de Informação Geográfica (SIG) de: (a) todos os municípios do mapa político do Estado da Bahia, com realce em amarelo para a localização do Território Litoral Sul; (b) a localização do Território Litoral Sul da Bahia com apresentação dos municípios, identificando-os por cores diferenciadas, e os (c) cinco municípios com os maiores índices de mortalidade infantil da região realçados na cor bege.
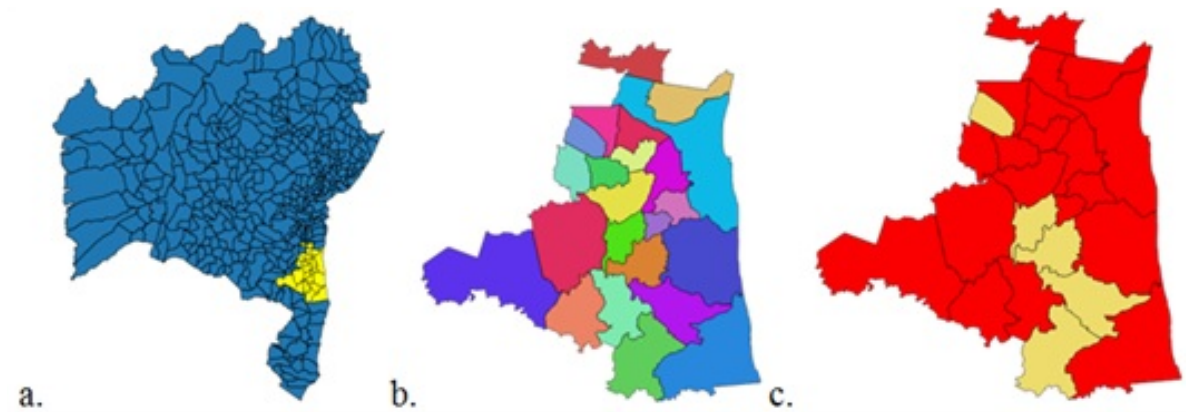

Figura 2 Mapas Georreferenciados apontando a Mortalidade Infantil no Sul da Bahia, 2018 Fonte: IBGE, 2010.

Verifica-se que, apesar dos municípios estarem em um mesmo território e com características epidemiológicas similares, existem peculiaridades para cada localidade. Um exemplo é o município de Itajú do Colônia que tem um dos maiores valores per capita $(\mathrm{R} \$ 125,52)$, porém apresenta a maior taxa de mortalidade infantil da região (35,17/ 1.000 nascidos vivos).

A partir de mapas georreferenciados visualizamos a proximidade dos municípios, bem como a necessidade de avaliar por que a rede de serviços não atinge a melhoria necessária para diminuir as altas taxas de mortalidade infantil nestas localidades. Esta questão é importante, visto que o serviço dentro do contexto do Sistema Único de Saúde deve ser administrado de forma que os 
municípios que têm mais condições (polos de serviços) vendam para aqueles que têm menos. Deste modo, é possível contemplar os princípios de universalidade e integralidade de saúde definidos constitucionalmente (9).

Por outro lado, na figura 3, mostra-se a representação gráfica da relação entre rendimento médio do trabalhador formal e os recursos da atenção básica distribuídos para o Território Litoral Sul como um todo, no tempo. De acordo com o gráfico, o conjunto dos usuários que recebem entre $\mathrm{R} \$ 900,00$ e R \$1.200,00 é o que mais utiliza o sistema público de saúde relativamente à atenção básica (R\$100,00 a R \$ 150,00 pessoa/ano, como consta no eixo horizontal da figura). Este dado corrobora a impressão mais geral do imaginário brasileiro de que o sistema público de saúde é "para pobres" (em oposição aos planos privados de saúde). Entretanto, a proposição da integralidade e universalidade dos serviços públicos de saúde é que o sistema deva atender a todos os cidadãos brasileiros, indistintamente.

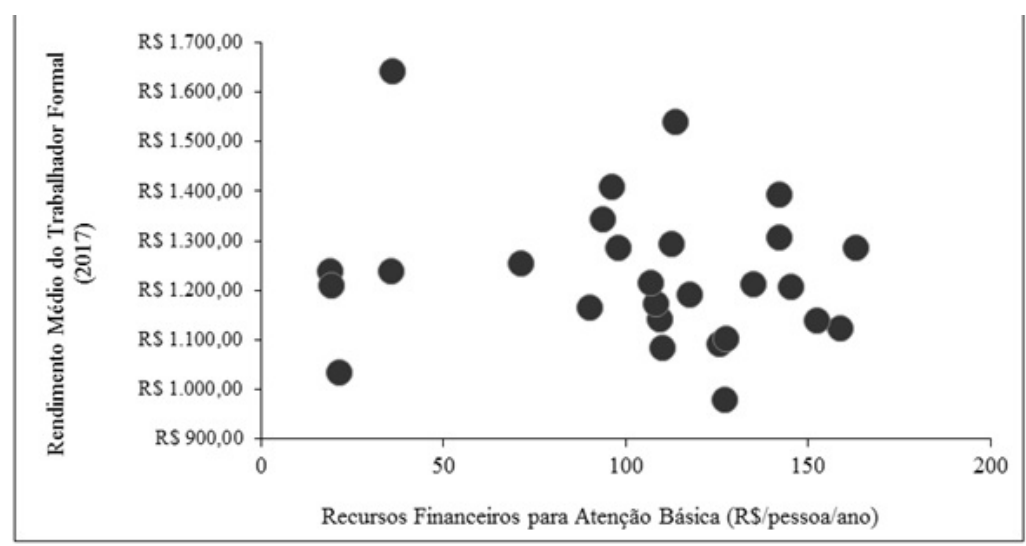

Figura 3 Rendimento médio do trabalhador formal e recursos financeiros da atenção básica da saúde, Sul da Bahia, 2017 Fonte: elaboração própria com base em dados do IBGE, 2010 e Datasus, 2018.

Embora não seja possível identificar aqui, é importante anotar o que diz a literatura especializada: o recurso financeiro para atenção básica alcança, em geral, aqueles com menores rendas, porém como está mal distribuída e tem uma gestão ineficiente, esta mesma população "consome" valores muito elevados com o cuidado em saúde. Por tal razão, em muitas ocasiões esta faixa é responsabilizada (em termos das finanças públicas) por inúmeros procedimentos quase sempre desnecessários (10).

\section{Discussão}

Os índices de mortalidade podem revelar muito a respeito da gestão da saúde pública e das condições sociais em um determinado lugar. Especialmente, eles podem determinar a relação entre a administração de recursos financeiros, o modelo de atenção à saúde e a qualidade de vida de uma população (11-14). Como indica Teixeira (15), realizar um levantamento das condições sociais e de saúde de uma determinada população significa revisar suas relações com as políticas públicas, produções científicas e sobretudo com o planejamento e a gestão em saúde (16). 
$\mathrm{O}$ indicador de mortalidade de menores de um ano de idade é considerado pela literatura especializada um dos mais sensíveis para medir e avaliar a situação de saúde e a condição social de uma dada população. Os óbitos de menores de um ano se relacionam diretamente com as condições de saneamento, habitação, educação, nutrição e assistência à saúde (principalmente no pré-natal e no parto). Assim, a taxa de mortalidade infantil é inversamente proporcional às condições socioeconômicas da população (4). Além dos fatores biológicos, os óbitos de menores de um ano se atrelam diretamente com as condições de escolaridade, raça e assistência à saúde (17).

A partir do uso dos mapas foi possível visualizar a contiguidade geográfica dos municípios com maior mortalidade infantil. Um dos motivos que poderiam explicar a semelhança epidemiológica entre os municípios de alta mortalidade infantil -excetuando-se os vetores sociais e econômicos- é a prioridade (ou não) do direcionamento dos recursos para o serviço materno-infantil na atenção básica (18).

A morbimortalidade infantil no Território Litoral Sul da Bahia representa as condições de vida e de saúde da sua população, uma vez que expressa causas biológicas, determinações de ordem ambiental, social e econômica, bem como os modelos de atenção e gestão que o sistema de saúde tem adotado para atender às necessidades regionais. Estes são instrumentos indispensáveis para definir as prioridades nas políticas públicas, planejar ações e serviços, monitorar a situação de saúde, bem como avaliar o desempenho do sistema em geral.

A análise da taxa média de mortalidade infantil no período de dez anos realçou a dimensão, em particular as crianças menores de ano, da vulnerabilidade da população residente no terreno investigado. Os mapas construídos com base no sistema de informação geográfica apresentaram a necessidade de avaliação em saúde no corredor de municípios que apresentam índices de mortalidade preocupantes. Por outro lado, o gráfico que relaciona o rendimento médio do trabalhador formal e os recursos financeiros da atenção básica da saúde (figura 3) demonstra como o alcance da resolutibilidade da atenção básica no território tem sido limitado.

Ao discriminar e calcular a taxa média de mortalidade infantil no período de uma década, verificou-se que mesmo os municípios polo e/ou referência em serviço de saúde, isto é, os municípios com maior população e melhor condição material e financeira, apresentam altas taxas de mortalidade. Tem-se, então, que este grave problema deve ser verificado a partir de uma perspectiva mais ampla, considerando os aspectos socioculturais, econômicos e ambientais mais gerais presentes no Território Litoral Sul.

Um olhar em perspectiva histórica mostra como a rede de sustentação social local traduz essencialmente políticas que não combatem às desigualdades sociais, mas que as reproduzem. Isso ocorre porque a área social não tem enfrentado de forma consequente, inclusive durante os governos progressistas, a reestruturação de sua lógica de financiamento e de atuação (19). 
Vale assinalar que a Constituição Brasileira (1988) institui o Sistema Único de Saúde (SUS) em seu artigo 196. O SUS tem sido reconhecido pela Organização Mundial de Saúde como o maior sistema gratuito e universal de saúde pública do mundo. A Carta Magna do Brasil estabelece, ainda, que "a saúde é direito de todos e dever do Estado, garantido mediante políticas sociais e econômicas que visem à redução do risco de doença e de outros agravos e ao acesso universal e igualitário às ações e serviços para sua promoção, proteção e recuperação" (9).

A lei brasileira define também que as ações e os serviços de saúde devem integrar uma rede regionalizada e hierarquizada de acordo com as diretrizes de descentralização, integralidade e participação social, sendo para isso financiada majoritariamente com recursos advindos da Seguridade Social, da União (Governo Federal), dos Estados e dos Municípios.

A descentralização do serviço de saúde é definida pela lógica de seu financiamento, estreitamente regulada e normatizada pelo governo federal em dois blocos: custeio e investimento. A despeito da sua dimensão colossal, o modelo do SUS enfrenta muitos desafios, incluindo várias contradições internas. A sua autonomia local está limitada e rigidamente controlada pela centralização do financiamento, que em última instância define planos, metas e o escopo das ações da saúde pública, incluindo o grau de complexidade por região e a política de pessoal (20).

Assim, para analisar o sistema de saúde no que se refere a gestão dos recursos financeiros é necessário levar em consideração alguns dos principais problemas do SUS. Estes são, esquematicamente, o financiamento ineficaz, um modelo de gestão fragmentado que opera em departamentos que não se comunicam (dificultando o uso intensivo e estratégico dos recursos existentes), e a inadequada política de pessoal para a saúde que vai desde a formação até a inexistência de uma carreira laboral fixada em lei.

Outro ponto de tensão diz respeito ao modelo de gestão da oferta do serviço de saúde cujo formato é o de prestador de serviço particular. Este vende seus serviços para o governo (uma quantidade específica de procedimentos médicos, de atendimentos, de exames de laboratórios por município, por exemplo). Trata-se da hegemonia de uma racionalidade empresarial que tende a invadir o espaço do SUS. Entretanto, este deveria funcionar segundo a premissa do serviço público integral (19).

O que realmente deveria ocorrer, segundo a lei, é uma gestão da saúde pública com foco na população, vinculada à atenção primária, classificando as famílias por nível sócio sanitário e por riscos biológicos, planejando e dando conta de todos os fluxos das pessoas ao longo da rede de saúde.

Em termos temporais, a distribuição dos recursos financeiros municipais relativos à Atenção Básica tornou-se instável com os governos liberais e privatistas da década de 1990. A partir da segunda metade daquela década as verbas passaram a ser contingenciadas (desviadas para finalidades diferentes daquelas definidas no orçamento original) em praticamente todos os níveis 
de governo, inclusive nos municípios. O Território Litoral Sul não foge do padrão nacional que registrou entre 1996 e 2011 uma redução significativa dos municípios no gasto público total com saúde, passando de $28,8 \%$ para $27,8 \%$ (22).

A Atenção Primária à Saúde/Atenção Básica em Saúde estabelece uma importante forma de acesso do usuário às ações e serviços do SUS, sendo considerada, apesar dos seus percalços, como prioritária por sua capacidade de resolver a maior parte das necessidades de saúde da população. Esse modelo de atenção está baseado no desenvolvimento de ações com alto grau de descentralização e adscrição ao território, estabelecendo vínculos com os usuários e a possibilidade de atenção integral. Isto se atinge a partir da identificação do grau de vulnerabilidade da população (10).

No âmbito do Território Litoral Sul da Bahia o dilema está entre sistemas fragmentados e sistemas integrados em redes de atenção à saúde. Os sistemas fragmentados, por falta de coordenação, são pouco eficientes e pouco efetivos, além de não estarem preparados para enfrentar doenças crônicas.

Assim, parece ser premente alinhar o modelo de financiamento, o modelo de atenção e o modelo de gestão de forma a abrandar as contradições internas do sistema. É um contrassenso que o modelo de atenção do SUS seja baseado, em tese, na promoção e prevenção da saúde, enquanto na prática se oferece um serviço de cariz curativista e de valor elevado para os cofres públicos. Isto representa um custo altíssimo para a população mais pobre, em termos de má qualidade e insuficiência do serviço prestado. É comum que o SUS apresente longas filas de espera, carência de insumos e medicamentos, o que representa um desafio à equidade como critério universal.

Vale ressaltar, no entanto, que mudanças apenas no âmbito do financiamento não são suficientes para equacionar o problema da atenção à saúde. Por si sós essas mudanças não mudarão o fato de que a mortalidade infantil seja um alarmante indicador de saúde pública no Brasil. Inclusive porque o SUS está inscrito no contexto de um país extremamente desigual.

Para Paixão e Ferreira (23), mudanças no sistema devem se dar concomitantemente e de forma alinhada nos três componentes dos sistemas de saúde: modelo político (de gestão), modelo técnico (de atenção à saúde) e modelo econômico (de financiamento). Mudando-se apenas o financiamento, isto é, aumentando o recurso, não vai se resolver o problema da atenção à saúde, e, por tanto, não será solucionado o problema da mortalidade infantil no contexto da saúde pública.

É preciso assinalar que dos municípios que compõem o Território Litoral Sul da Bahia, mais de $80 \%$ apresentam um índice de mortalidade infantil acima da média nacional em todo período estudado. A variação deste índice no fator tempo está fortemente correlacionada com fatores socioeconômicos e ambientais. 
Este trabalho se constitui como uma forma de contribuir para o entendimento dos indicadores de mortalidade infantil no âmbito do financiamento da saúde. Deste modo, é possível visualizar os nós críticos relativos à qualidade de vida da população, ressaltando que o fortalecimento da atenção básica e a descentralização dos serviços do SUS são de extrema importância para a melhoria do acesso à saúde no Brasil. Reitera-se, ainda, a necessidade de investigações mais aprofundadas que contribuam para um desenho mais completo e preciso da saúde pública no contexto brasileiro.

O estudo aqui apresentado procurou demonstrar como ações e serviços no campo da saúde pública devem contemplar realidades mais específicas que permitam certas mediações entre o local e o geral. Isto é, mediações entre os contextos socioeconômicos e ambientais concretos e singulares, e os modelos mais gerais de atenção à saúde, desenhados para atender a todo um país. Deste modo, podem se plasmar políticas, instituições e práticas de saúde em um arranjo funcional e eficiente.

\section{Referências}

1. Kaur P, Singh S. A Social autopsy to identify the socioeconomics dimension and determinates of infant mortality in Haryana. Journal of the Gujarat Research Society. 2019;21(13): 260-272. http:/gujarat researchsociety.in/index.php/JGRS/article/view/1257

2. Wirsiy FS, Ako-Arrey DE, Yeika EV. Infant mortality in the Central African Region: A time trend descriptive analysis. Journal of Pediatrics, Perinatology and Child Health. 2019;3(4): 201-207. http://www.fortunejournals.com/articles/infant-mortality-in-the-central-african-region-a-ti me-trend-descriptive-analysis.html

3. Lu Z, Bandara JS, Paramati SR. Impact of sanitation, safe drinking water and health expenditure on infant mortality rate in developing economies. Australian Economic Papers. 2020;59(1): 13-33. https://do i.org/10.1111/1467-8454.12167

4. WHO. World Health Organization. Levels and trends in Child Mortality 2017. Geneva. 2017. http://www .crianca.mppr.mp.br/arquivos/File/publi/unicef_relatorios/child_mortality_report_unicef_2017.pdf .

5. Careti CM, Furtado MCC, Barreto JC, Vicente JB, Lima PRL. Ações em saúde na Atenção Básica para redução da mortalidade infantil. Rev RENE. 2016;17(1): 67-75. http://periodicos.ufc.br/rene/article /view/2607

6. BRASIL. Ministério da Saúde. [base de dados Internet]. Sistema de Informação de Agravos de Notificação (SINAN). Departamento de Informática do Sistema Único de Saúde do Brasil (DATASUS). http://datasus.saude.gov.br

7. BRASIL. Ministério da Saúde. Sistema de Informações sobre Orçamentos Públicos em Saúde (SIOPS) Departamento de Informática do Sistema Único de Saúde do Brasil (DATASUS). https://datasus.sa ude.gov.br/sistemas-e-aplicativos/financeiros/siops.

8. IBGE. Instituto Brasileiro de Geografia e Estatística. Cidades e Estados, 2019. https://www.ibge.gov.br/. 
Alciene Pereira da Silva/ Roque Pinto/ Lacita Menezes Skalinski/ Alisson Jadavi Pereira da Silva/ et ai.

9. Brasil, Senado Federal. Constituição da República Federativa do Brasil: texto constitucional promulgado em 5 de outubro de 1988, com alterações determinadas pelas emendas Constitucionais de revisão n. 1 a 6/94, pelas emendas Cosntitucionais n. 1/92 a 91/2016 e pelo Decreto Legis. Cosntituição da República Fed do Bras. 2016;1:496. https://www2.senado.leg.br/bdsf/bitstream/handle/id/518231/C F88_Livro_EC91_2016.pdf?sequence=1

10. Mendes EV. O cuidado das condições crônicas na atenção primária à saúde: $O$ imperativo da consolidação da estratégia da saúde da família. Brasília: Organização Pan-Americana da Saúde; 2012.

11. Cerqueira CA. Políticas públicas de desenvolvimento territorial rural: uma análise da delimitação dos territórios rurais do estado da Bahia, segundo a tipologia municipal. Tese de Doutorado. Uberlândia: Universidade Federal de Uberlândia; 2015.

12. Giovanella L, Almeida PF de, Vega RR, Oliveira S, Tejerina SH. Panorama de la Atención Primaria de Salud en Suramérica: concepciones, componentes y desafíos. Saúde debate. 2015;39(105): 300-322. https://www.scielosp.org/article/sdeb/2015.v39n105/300-322/es/

13. Ursache, SA, Muntele, I. Factorial Analysis of the Dynamics of Infant Mortality in Romania (2001-2017). Case Study: Iaşi County. Rom J Populn Stud. 2019;23(2): 101-117. https://www.ceeo 1.com/search/article-detail $\mathrm{id}=838634$

14. Raghupathi V, Raghupathi W. The influence of education on health: An empirical assessment of OECD countries for the period 1995-2015. Arch Public Health. 2020;78(20): 1-18. https://doi.org/10.1186 /s13690-020-00402-5

15. Teixeira CF. Planejamento municipal em saúde. Instituto de Saúde Coletiva; 2001.

16. Souza Júnior MA, Mendes A. Efeitos das políticas econômicas restritivas sobre a condição de saúde dos brasileiros. JMPHC, 2020;12: 1-32. https://doi.org/10.14295/jmphc.v12i.971

17. Maia LTS, Souza WV, Mendes ACG. Determinantes individuais e contextuais associados à mortalidade infantil nas capitais brasileiras: uma abordagem multinível. Cad Saúde Publica. 2020;36(2): e00057519. https://doi.org/10.1590/0102-311x00057519

18. Brasil. Lei no 8.080, de 19 de setembro de 1990. Lei Orgânica da Saúde. Dispõe sobre as condições para a promoção, proteção e recuperação da saúde, a organização e o funcionamento dos serviços correspondentes e dá outras providências. Brasília; 1990.

19. Campos GWS, Lima LD, Rizzotto MLF, Lobato LVC, Luiza VL, Mattos RA Entrevista com o Professor Gastão Wagner de Sousa Campos. Saúde em Debate. 2015;39(spe): 338-339. https://www.scielo.br /scielo.php?pid=S0103-11042015000500338\&script=sci_arttext

20. Brasil. Ministério da Saúde. [base de dados Internet]. Portaria 2.436/2017. Política Nacional de Atenção Básica, estabelecendo a revisão de diretrizes para a organização da Atenção Básica, no âmbito do Sistema Único de Saúde. Brasília; 2017.

21. Brêtas Júnior N, Mendes A, Matheus E, Rinehart D, Carvalho GC, et al. A atenção básica que queremos. CONASEMS - Cons Nac Secr Munic Saúde. 2011;43. http://www.cosemsam.org.br/wp-content/up loads/2016/04/a-atencao-basica-que-queremos.pdf 
Mortalidade infantil e orçamento público. Desafios na gestão do sistema de saúde no Território Litoral Sul da Bahia, Brasil

22. Araújo CEL, Gonçalves GQ, Machado JA. Os municípios brasileiros e os gastos próprios com saúde: Algumas associações. Cienc e Saude Coletiva. 2017;22(3): 953-963. https://doi.org/10.1590/141381232017223.15542016

23. Paixão AN, Ferreira T. Determinantes da mortalidade infantil no Brasil. Inf Gepec. 2012;16(2): 6-20. h ttp://e-revista.unioeste.br/index.php/gepec/article/view/3967

Notas

* $\quad$ Artigo de pesquisa científica.

1 O Território Litoral Sul do Estado da Bahia é formado pelos seguintes municípios: Almadina, Arataca, Aurelino Leal, Barro Preto, Buerarema, Camacan, Canavieiras, Coaraci, Floresta Azul, Ibicaraí, Ilhéus, Itabuna, Itacaré, Itajú do Colônia, Itajuípe, Itapé, Itapitanga, Jussari, Maraú, Mascote, Pau Brasil, Santa Luzia, São José da Vitória, Ubaitaba, Una e Uruçuca. 